\title{
Philosophiques
}

\section{Espace et intelligilité mathématiques : sur le problème mathématique de l'espace}

\section{Alain Michel}

Volume 24, numéro 2, automne 1997

URI : https://id.erudit.org/iderudit/027458ar

DOI : https://doi.org/10.7202/027458ar

Aller au sommaire du numéro

Éditeur(s)

Société de philosophie du Québec

ISSN

0316-2923 (imprimé)

1492-1391 (numérique)

Découvrir la revue

Citer cet article

Michel, A. (1997). Espace et intelligilité mathématiques : sur le problème mathématique de l'espace. Philosophiques, 24(2), 349-366.

https://doi.org/10.7202/027458ar d'utilisation que vous pouvez consulter en ligne.

https://apropos.erudit.org/fr/usagers/politique-dutilisation/ 


\title{
ESPACE ET NIELLIGIBIIIIÉ MATHÉMATIOUES : SUR IF PROBLĖME MATHÉMATIOUE OF L'ESPACE
}

\author{
PAR \\ ALAIN MICHEL
}

L'ouvrage de M. Boi est impressionnant à plus d'un titre, mais d'abord par son ampleur. À vrai dire, une telle ampleur était exigée par le problème traité, celui de l'espace, un des plus vastes, des plus profonds, des plus riches de perspectives, de toute la philosophie des sciences. Comme l'auteur le remarque lui-même, citant $\mathrm{R}$. Torretti et $\mathrm{E}$. Scholz, aucun ouvrage n'existait encore qui fût d'une envergure suffisante pour prétendre constituer un traitement digne du sujet. M. Boi a voulu au fond écrire le livre qui aurait pu, au moment où il entreprenait ses recherches, le satisfaire. Le résultat est à la hauteur des espérances, et l'on trouve dans l'ouvrage un reflet de la grandeur du sujet. Oserons-nous enfin nous féliciter de ce qu'un ouvrage destiné à faire référence soit écrit en français, et n'hésite pas à citer des philosophes français des mathématiques: A. Lautman, J.-T. Desanti, G. Granger, J. Vuillemin?

Sa richesse est double. Elle est en premier lieu dans la variété des thèmes envisagés, qui se rapportent sans doute tous, directement ou indirectement, à l'espace, mais qui sont très divers.

On trouve d'abord des thèmes proprement géométriques. Au fondement de tout, il y a bien sûr la découverte des géométries non euclidiennes, avec la construction des différents systèmes de ces géométries. C'est ce que l'auteur appelle le passage, en géométrie, de l'univers unique à la pluralité des mondes possibles. Il implique la rupture par rapport à la conviction que l'espace euclidien était le seul géométriquement possible, en même temps que le cadre même des phénomènes naturels.

Le deuxième événement, qui, s'il n'est pas la suite directe du précédent, est étroitement lié à lui (un point rarement évoqué), consiste dans l'émergence d'une nouvelle conception des êtres géométriques : c'est la géométrie intrinsèque de Gauss, résultat de l'élaboration de la théorie des surfaces courbes.

M. Boi montre que ces deux événements sont la source même du problème de l'espace en tant que problème mathématique authentique : d'abord parce qu'on le distingue conceptuellement de l'espace sensible ou physique ; ensuite parce que c'est alors seulement qu'on conçoit que l'espace lui-même, en tant que tel, peut être doté d'une structure 
mathématique, et de propriétés géométriques. De ces événements décisifs suivront la notion de géométrie sur une variété, avec les deux concepts clés de courbure et de torsion, et tout le cortège de ses conséquences proprement mathématiques sur le développement de l'algèbre (calcul tensoriel), de l'analyse et de la géométrie différentielles (transport parallèle, connexion, ...), la théorie des formes spatiales, etc.

On rencontre ensuite le thème, non moins riche, des rapports entre géométrie et physique. Déjà avec Gauss, mais surtout à partir de Riemann, se trouve posée la question des rapports entre la géométrie et l'espace physique - de manière générale celle des rapports entre espace, continu et matière (et notamment des relations entre la métrique et la matière émergence de l'idée d'un conditionnement de la première par la seconde) ; la théorie spatiale de la matière de Clifford (identification pour l'essentiel de l'espace à la courbure, théorie de la courbure-matière), dont l'examen forme le chapitre final du livre.

Au fil de la lecture, on trouvera abordés aussi des problèmes historiques: l'évaluation de l'importance et du rôle historiques de la découverte, précédemment évoquée, des géométries non euclidiennes, l'analyse du mémoire de Riemann, restitué dans son contexte historique (l'influence de Helmholtz) et des interprétations, jugements, appréciations, quelquefois adverses, auxquels il a donné lieu; mais aussi des problèmes qu'on qualifie généralement de "philosophiques ": existe-t-il quelque chose comme une philosophie de l'espace ? à quelles conditions ? est-il légitime de réanimer ce qui avait été l'ambition de la Naturphilosophie?

Un autre aspect de la richesse de l'ouvrage est la multiplicité des points de vue auxquels l'auteur se place, en nous invitant à le suivre.

Le premier par ordre d'importance nous paraît être le point de vue historique. À l'évidence, il forme le fil directeur du livre : dès la page XIII, délimitant son projet, l'auteur explique qu'il s'agira d'un " approfondissement historique ", d'une étude des a développements conceptuels ", des " modes de formation " des notions fondamentales, d'une " approche généalogique ». De fait, l'ouvrage se présente comme une suite d'épisodes de l'histoire de la géométrie : découverte des géométries non euclidiennes, notamment chez Bolyai et Lobatchevski ; mise au point, à partir de là, dans une continuité historique, de la géométrie intrinsèque des surfaces, avec Gauss ; analyse de l'œuvre de Riemann (avec l'émergence du concept de variété, et les débuts de la géométrie différentielle moderne), qui forme vraiment la partie centrale du livre, interprétation des géométries non euclidiennes sur des volumes de l'espace euclidien par Beltrami, avec les développements de géométrie infinitésimale et différentielle qui s'ensuivent, élaboration enfin du concept de variété, avec ses conséquences chez Helmholtz et dans la géométrie « elliptique » de Clifford. 
Cette histoire est largement et précisément traitée, avec de substantielles citations des textes originaux, des analyses et des commentaires développés jusque dans le détail, un grand luxe de précisions chronologiques, biographiques, bibliographiques, et même l'inclusion d'écrits inédits (correspondance d'E. Beltrami et de J. Houël sur le modèle pseudosphérique de la géométrie non-euclidienne).

C'est d'autre part un des traits les plus remarquables de l'ouvrage, et son originalité par rapport aux ouvrages purement historiques, que le point de vue réflexif n'y soit jamais délaissé, mais qu'au contraire les analyses historiques y reçoivent le constant support de la réflexion philosophique ou épistémologique. En fait, tous les chapitres incluent un développement épistémologique ou philosophique : voir, dans le chapitre premier, la philosophie de la géométrie de Lobatchevski; dans le chapitre II, le développement sur géométrie, physique et métaphysique de l'espace chez Gauss (2-6) ; dans le chapitre III, les remarques épistémologiques à propos de Riemann (3-6), ou de l'interprétation de Beltrami (6-5), ou encore de la découverte des géométries non euclidiennes en général (8-1). Des questions généralement laissées de côté par les historiens ou philosophes des mathématiques sont traitées : critique de Husserl à Riemann; conceptions de Helmholtz, critiques adressées à Helmholtz par des commentateurs plus ou moins obscurs : Ueberweg, Delbøuf, Houël, Tilly...

Cette variété des thèmes abordés et des points de vue auxquels on peut les aborder est moins favorable au commentateur, qui hésite légitimement dans le choix des thèmes de réflexion. Nous avons choisi de privilégier le point de vue disons philosophique, peut-être plus difficile d'accès, mais qui nous semble plus propre à permettre de dégager les lignes de force et les enjeux réels, et à nous garder de nous perdre dans le détail des reconstitutions et interprétations historiques, en nous contraignant à envisager les données sous l'angle des problèmes qui s'y trouvent implicitement posés.

Les titre et sous-titre du livre délimitent son champ et indiquent son ambition. D'abord il s'agit du problème mathématique de l'espace -- et non du problème physique, encore moins biologique ou psychologique, même si l'auteur ne pouvait se désintéresser des rapports entre l'espace géométrique et l'espace physique, ou, plus généralement, des relations entre la géométrie et la physique. Mais le sous-titre, " une quête de l'intelligible ", signifie clairement qu'il s'agit aussi de philosophie. L'auteur défend des thèses propres, et on sent bien que, sur un sujet aussi vaste, elles ont orienté ses choix et l'allure des développements. Il me semble qu'on pourrait les résumer ainsi, en les regroupant.

Quant au statut général des objets et de la connaissance mathématiques, l'auteur a visiblement beaucoup de sympathie pour l'idée d'une nature qualitative et spatiale des mathématiques. Historiquement, cette idée 
s'enracine dans l'œuvre de Gauss, montrant la nature intrinsèque et géométrique des entités mathématiques, mais elle forme le fond de la conception riemannienne, essentiellement géométrique, de la variété, dans sa liaison même au concept fonctionnel de " surface de Riemann ". Mais sa signification dépasse de loin, me semble-t-il, les traces qu'elle a laissées dans l'histoire, même si les épisodes rappelés sont d'une importance décisive, importance dont le livre sait si bien nous convaincre.

En ce qui concerne l'interprétation de la connaissance mathématique, l'auteur privilégie visiblement, en consonance avec le privilège accordé à la spatialité qualitative, le rôle de l'intuition, de la vision synthétique, dans l'élaboration des notions. L. Boi tient à se démarquer ici de l'école du positivisme ou de l'empirisme logiques dont il rejette nettement les thèses sur la réductibilité des mathématiques à un langage formel : voir p. 396, à propos de Helmholtz; ou encore, plus loin, p. 402, la critique du dogme traditionnel selon lequel le seul procédé permettant d'établir une proposition scientifique consisterait à s'appuyer sur la logique ou sur l'expérience, si bien que toutes les vérités seraient, soit de nature logique, soit de nature empirique. Et c'est justement ce qui caractérise la conception de Helmholtz. Il est sans doute significatif à ce propos que les recherches de Carnap sur l'espace ne soient pas - ou très incomplètement - citées : notamment un des premiers, sinon le premier écrit du jeune Carnap, antérieur à l'Aufbau, de 1928, Der Raum, ein Beitrag zur Wissenschaftslehre, Berlin, 1922, même date que le manuscrit Vom Chaos bis Wirklichkeit, prélude de l'Aufbau). Il n'adopte pas pour autant toutes les tendances de l'école française, notamment celles de la tradition de Cavaillès, représentée en France par des auteurs comme J. Desanti, G. Granger, J. Vuillemin, qui sont tous cités à des degrés divers, quoique sans esprit d'allégeance.

Sur ces deux points, des questions, ne serait-ce que d'éclaircissement, s'imposent. Quant au problème plus spécifique de l'espace, il me semble que le centre de l'étude, qui agit comme le foyer en quelque sorte des conceptions de l'espace exposées dans le livre, sans doute parce qu'elle les domine, jusque dans leurs aspects les plus contemporains, est l'œuvre de Riemann. Il faudrait citer, comme accompagnement presque indispensable, les commentaires de H. Weyl. D'abord parce que ce dernier est le promoteur de la théorie des variétés, décisive pour la mise au point du concept moderne d'espace, à l'âge des géométries non euclidiennes. Ensuite parce qu'il a été à l'origine du mouvement de géométrisation de la physique qui a trouvé son point d'aboutissement dans la théorie de la relativité générale. L'espacetemps de la théorie est une variété riemannienne à quatre dimensions l'interprétation géométrique de l'espace est commune à $\mathrm{H}$. Weyl, E. Cartan et Einstein lui-même. Là aussi l'interrogation peut venir. On peut en dire tout de suite quelques mots, car cela ne touche pas à l'essentiel du propos de l'auteur. 
L'auteur lui-méme le reconnaît : ce qu'il propose est une lecture de l'ceuvre de Riemann, et cette lecture s'alimente à la tradition de la Naturphilosophie. M. Boi annonce d'ailleurs, incluse dans un ouvrage d'ensemble, une traduction commentée des Fragmente philosophische Inhalts, des fragments posthumes de Riemann (édités dans la première édition des œuvres de Riemann par Dedekind et Weber), dont la troisième et dernière partie porte justement sur la Naturphilosophie.

Le chapitre III contient une analyse précise du contexte historique, avec, notamment, la restitution des commentaires auxquels a donné lieu l'œuvre riemannienne : voir la mise au point de la note 97 , p. 183, et le rappel des critiques du mémoire de Riemann, après Husserl, Brentano, par Lotze, Stallo, etc., aux pp. 251 et ss. Un problème assez souvent débattu ici est celui de l'influence de Herbart. L'influence de Gauss ne pose pas les mêmes problèmes, et elle est nettement plus probable : d'abord parce qu'il est mathématicien, et des plus grands, ensuite parce qu'il s'intéresse aux mêmes choses, enfin parce qu'on sait qu'il a été, sinon le maître, du moins l'examinateur de Riemann. L'auteur rappelle (pp. 132-ss, 211-ss) que Russell niait l'influence de Herbart. E. Scholz, qui reprend la question après examen des textes, confirme en gros cette opinion. Je pencherai personnellement en faveur de cette minoration. M. Boi affirme, p. 134, que " chez lui, la recherche mathématique proprement dite s'accompagne toujours d'une réflexion philosophique à propos du statut que possèdent les concepts mathématiques abstraits et du rôle qu'ils jouent quant à l'explication des phénomènes naturels ». L'appréciation de ce qui est philosophique et de ce qui ne l'est pas est sujette à variation, et, sous cette forme, on pourrait effectivement cautionner la thèse. Il ne faudrait pas pour autant lui donner trop d'importance dans l'analyse de l'œuvre de Riemann, et c'est ce que L. Boi ne fait pas. Riemann est essentiellement mathématicien, et les influences éventuelles sont d'abord de nature mathématique. Il est hors de question de nier, par exemple, le bien-fondé de l'affirmation selon laquelle Riemann s'intéresse à une généalogie des concepts, et qu'il refuse à la fois, en ce qui concerne l'espace, l'idée d'une origine empirique (dans le réel sensible) et d'une origine mentale (dans le réel psychologique). On ne saurait trop louer ici la prudence de l'auteur, son effort pour séparer les genres, son souci de ne rien affirmer qu'il ne puisse justifier par la référence à des textes précis. Ses conclusions sur le concept riemannien de l'espace sont parfaitement nettes : 1) il n'est pas de nature psychologique ; 2) il s'agit déjà du concept moderne ; 3 ) il enveloppe des structures mathématiques essentielles : métrique et différentiable, qui porteront le fruit mathématique que l'on sait. On peut laisser le reste à la reconstruction du psychologue : du moment où on dispose de son œuvre, on n'a nul besoin - et l'épistémologue n'a que faire - de savoir ce qui s'est passé dans l'esprit de Riemann.

Venons en aux problèmes plus généraux soulevés par le livre. Le premier est celui des rapports entre philosophie et mathématiques. Il est abordé aussi 
à propos de Husserl (pp. 244-ss), qui rejette ce qui a constitué la rupture épistémologique de Riemann : faire passer la pensée géométrique du règne des lois à celui des structures, au profit d'une réhabilitation de la géométrie euclidienne comme seul langage naturel quoique (et parce que) purement idéal. D'après L. Boi, la différence essentielle entre le philosophe et le mathématicien est que, alors que le second se préoccupe surtout de créer de nouvelles structures et de nouveaux objets, le premier cherche à rattacher ses idées à une tradition ou une généalogie, et à montrer comment ils tirent leur sens d'une pensée antérieure. Il y a peut-être quelque abus à transformer ainsi le philosophe en historien de sa propre pensée, en une sorte d'“ antiquaire ", selon l'expression de Nietszche dans les Considérations inactuelles. Mais on peut tomber d'accord sur le constat de l'incompréhension décevante manifestée par Husserl. Confrontant ces textes avec d'autres plus tardifs, moins dogmatiques, l'auteur repêche finalement Husserl. On serait tenté d'être moins bienveillant, et de penser que l'erreur de Husserl était inévitable, déjà inscrite dans son plus vieux souci : celui de réassigner à la philosophie un projet radical, aussi radical, selon les Méditations cartésiennes, que celui de Descartes. Cavaillès a montré la présence, dans la Krisis..., du thème de l'utopie de l'archéologie fondatrice, du mythe du retour aux « évidences primordiales " : l'histoire n'étant en mesure de révéler les sens authentiques que dans la mesure où elle permet de retrouver les liens perdus ${ }^{1}$. C'est bien ce que M. Boi met en évidence à propos de la critique husserlienne de Riemann, et de l'usage qu'il fait du concept de variété ${ }^{\text {. }}$ Husserl y affirme une primauté de la géométrie euclidienne, présupposée selon lui dans toute tentative de construction d'espaces généralisés. On ne peut définir une surface ou une variété de dimension supérieure à deux en faisant abstraction de l'espace euclidien à trois ou plus de trois dimensions, car l'espace euclidien représente le cadre naturel et absolu de la théorie des surfaces et variétés. L'espace euclidien est primordial; la géométrie euclidienne n'est pas une donnée contingente, mais elle est inscrite dans la nature même de l'espace, à laquelle elle appartient comme une a donnée originaire ${ }^{3} n$.

Le second problème est celui de la nature et du statut de la connaissance et des objets mathématiques. On n'aura pas trop de scrupule à utiliser ici, pour essayer de voir plus clair dans les positions de l'auteur, un texte dont l'essentiel se trouve dans les notes puisque lui-même nous a averti en

1. Sur la logique..., p. 77-ss. "Le retour à l'origine est retour à l'original „, et, si l'histoire empirique est utilisée comme révélateur des enchainements authentiques, c'est à l'envers, par le mythe du retour au passé... Rappelons que, selon Cavaillès, ces spéculations laissent intact le problème principal d'une épistémologie mathématique : celui de rendre compte du progrès comme tel, du progrès effectif, avec les structures et les entités qui le jalonnent.

2 Cours du semestre d'hiver 1889-1890, intitulé Coup d'ail sur le développement historique de la géométrie, publié par I. Strohmeyer et M. Nijhoff en 1983 dans le XXI' volume des Husserliana, Studien zur Arithmetik und Geometrie.

3. Dans sa critique la plus intéressante, Husserl montre qu'il faut distinguer entre variétés courbes, ou à courbure, et variétés tordues, ou à torsion (p. 235-239,243). 
introduction que les notes constituaient un autre texte, un para-texte essentiel à la compréhension. Dans les pages 396 et ss., l'auteur s'explique clairement à ce sujet. Il s'agit de l'examen critique des positions de Helmholtz. Les espaces abstraits construits par le mathématicien à la suite de la révolution non euclidienne sont susceptibles d'une représentation intuitive, mais non pas perceptive ou sensorielle. Ils expriment, dit l'auteur, des contenus mathématiques précis, et ces contenus ont une signification "objective ". Celle-ci ne saurait être cherchée, ni du côté des faits, ni du côté des énoncés logico-déductifs qui les expriment. Elle n'est ni formelle ni empirique. Cela paraît évident pour les faits empiriques. À propos du formel, l'auteur donne l'argument suivant: s'il était vrai que les contenus de signification étaient réductibles au langage formel ayant permis de les fixer, on ne pourrait comprendre qu'un même concept ou résultat puisse être exprimé de plusieurs façons équivalentes : par exemple à la fois sous une forme analytique et algébrique (on en aurait de nombreux exemples dans la théorie de l'espace).

L'auteur fait ici référence à la description que donne Desanti de l'objet mathématique comme idéalité : noyau permanent dans la variabilité et pôle de réactivation, celle-ci étant potentiellement indéfinie. Cette description s'inscrit cependant, chez Desanti, dans une caractérisation plus large et plus profonde de la production des objets mathématiques. Il s'agit, en mathématiques, d'apprendre à penser comme réels des sortes d'objets qui ne sont accessibles que dans le système des possibilités réglées ouvertes par les relations qui les définissent. Une idéalité mathématique n'est en ce sens rien d'autre qu'une indication de procédure opératoire ou démonstrative. Sans doute, seule une procédure d'écriture symbolique (et le plus souvent purement idéale) fixe ces sortes d'objets, par des systèmes infinis d'architectures de signes, pour lesquels on peut définir des codes explicites de désignation. Mais les objets n'existent pas en dehors des systèmes de relations où s'inscrivent leurs propriétés : leur mode d'existence est en ce sens entièrement intra-théorique, relatif aux propriétés du système dans lequel ils sont accessibles. L'idéalité-objet à laquelle renvoie toujours le signe mathématique doit alors être maintenue comme thème de vérifications concordantes - et elle renvoie elle-mème à une idéalité-champ, reproduite comme domaine de connexion des systèmes de vérification exigés.

De ces analyses, l'auteur ne retient guère que la thèse de l'objectivité des significations. Ainsi la signification des espaces géométriques (mais on peut penser que la thèse de l'auteur est ici générale et qu'il pense aux mathématiques dans leur ensemble) doit être cherchée dans l'ensemble des objets : concepts, structures, théories et méthodes qui président à la constitution de l'espace. Ces contenus représentent l'univers objectif des mathématiques, c'est l'ensemble de leurs contenus de connaissance. Sous cette forme, la thèse est également assez proche (à une nuance près sur laquelle nous allons revenir) de celle que soutient G. Granger concernant ce 
qu'il appelle des a contenus formels ", d'où sans doute le besoin que ressent M. Boi de s'expliquer dans une note. Un contenu de connaissance lui semble désigner une réalité plus profonde que celle d'un contenu formel au sens de Granger. Mais il ne s'explique là-dessus que sur un exemple. Celui qu'il prend est relatif aux groupes de transformations : le fait que ces groupes aient un certain nombre de paramètres plutôt qu'un autre relève des contenus formels; le fait qu'il soit continu ou discret relève des contenus de connaissance. La raison semble en être que la propriété de continuité est jugée plus fondamentale que le paramétrage : c'est « un concept constitutif des mathématiques ". Ainsi la description de l'activité mathématique comme productrice de contenus formels laisserait échapper le plus fondamental. On comprend, à la lecture de l'ouvrage, que M. Boi n'a qu'un penchant modéré pour les disciplines formelles. On peut partager cet éloignement, tout en se demandant si, en l'espèce, il ne le conduit pas à sous-estimer la portée de la notion proposée par M. Granger. Il semble en effet qu'il y ait malentendu, et que celui-ci porte sur l'expression “ formel ^. À notre sens, une telle expression n'implique pas l'idée d'un arbitraire dans un choix, comme, dans l'exemple pris, le choix d'une valeur pour des symboles (jouant le rôle de coefficients) donnés. Telle que l'entend Granger, elle est beaucoup plus générale, et s'étendrait, semble-t-il, sans difficulté jusqu'à cette notion fondamentale qu'est en effet la continuité. Il en irait de même pour les deux autres exemples pris par l'auteur, celle de la conception de l'espace comme une variété continue et différentiable, et celle de l'ensemble des mouvements de l'espace euclidien comme groupes de transformations. Dans l'expression " contenu formel ", "formel "est là pour souligner la nature fondamentalement symbolique du concept, comme de la connaissance, mathématique, et même de toute connaissance d'objet en général. Il s'agit, semble-t-il, d'éviter l'interprétation essentialiste du concept, sans glisser pour autant dans une dérive nominaliste (il lui arrive ainsi de parler d' universaux du langage ", mais par prétérition, en introduisant immédiatement une mise en garde). Granger insiste bien à ce propos sur la nécessité d'éviter le double piège d'une interprétation empiriste et d'une interprétation essentialiste des concepts mathématiques. D'une part, ils ne sont pas dérivables du sensible et de la perception, comme suffirait à l'attester le développement prodigieux de l'univers mathématique, son détachement systématique de la saisie des objets sensibles. Il convient de prendre les objets mathématiques en eux-mêmes, et de considérer au contraire les formes perceptives comme des applications, sans pour autant nécessairement les situer postérieurement dans une genèse ou une chronologie. D'autre part, il ne s'agit pas non plus d'interpréter ces concepts comme des essences idéales situées dans un arrière-monde, mais comme objectivations de propriétés de systèmes de symboles, des concrétisations de formes constructives d'expression : produits par un travail jamais achevé, dont les différents moments relèvent d'une dialectique 
interne, que le philosophe peut s'appliquer à reconstituer, à travers les cuvres de la mathématique.

M. Boi se réfêre volontiers à Kant dont il cite avec faveur, se déclarant en accord sur le fond, plusieurs interprétations récentes, notamment celles de MM. Petitot ou Salanskis. On peut effectivement penser qu'une réflexion sérieuse sur l'espace ne peut éviter, au moins comme point de départ, la référence à Kant. On tomberait volontiers à ce propos d'accord avec l'auteur sur le fait qu'une réhabilitation du kantisme, si elle est souhaitable, ne saurait en concerner qu'une forme partielle et locale. C'est surtout, selon lui, l'idée centrale de l'esthétique, selon laquelle "la géométrie est une science qui détermine synthétiquement et pourtant a priori les propriétés de l'espace ", qui s'est trouvée confirmée par les développements récents de la géométrie (p. 318). C'est elle qui garde sa validité. Mais son contenu doit être réélaboré pour tenir compte précisément du développement des géométries non euclidiennes et de la relativité générale.

Les études épistémologiques relatives à l'espace s'organisent en gros autour des trois points de vue suivants :

1) un point de vue psychologique et empirique : celui de la détermination des propriétés spatiales de la perception. Le problème est ici de construire un modèle abstrait de l'espace perceptif, avec la nécessité de définir un référentiel, distinct des autres référentiels éventuels. C'est un problème psychologique.

2) un point de vue physique : le cadre des phénomènes est défini par une physique dont les objets ne sont pas seulement, et directement, atteints par la perception. Le problème est alors, pour une méta-théorie et une épistémologie de la théorie physique, celui de la détermination du cadre spatial des objets physiques. La géométrie donne le cadre d'une représentation d'un mondè dans une totalité sensible et intersubjective : comment se fait-il que la multiplicité des points de vue du sujet soit compatible avec l'invariance de certains traits des phénomènes (objectivité de la physique)?

3) enfin, le point de vue proprement mathématique : quelle est la nature du concept mathématique d'espace?

Soit l'exemple de Poincaré. On retrouverait facilement, dans les études qu'il a consacrées au fondement de la géométrie et de notre notion d'espace, ces trois points de vue, même si chez lui l'approfondissement du premier et du troisième conduit en fait à dénier toute légitimité au deuxième. Avec le problème consistant à expliquer l'applicabilité de différents systèmes, euclidiens et non euclidiens, à notre espace et celui de savoir pourquoi on dit que notre espace a trois dimensions, le problème de l'explication des origines de nos idées fondamentales d'espace et de géométrie est un des problèmes fondamentaux de Poincaré. C'est ainsi que son étude de 1898, Sur les fondements de la géométrie, comporte, à côté d'une partie proprement 
mathématique, toute une partie empirique et génétique, où l'on trouve développées les implications de la question : comment obtenons-nous notre connaissance de l'espace et de ses propriétés? Il s'agit d'une théorie de la genèse de nos idées d'espace et de notre connaissance de la géométrie spatiale, qui constitue à coup sûr une expression importante de son conventionnalisme ${ }^{4}$. Poincaré y donne une explication psychophysiologique de l'origine de notre concept mental d'espace, et de la géométrie correspondante. La question est traitée d'un point de vue qu'on peut dire subjectiviste, ou même inter-subjectiviste ${ }^{5}$. Comment chacun de nous obtient-il une connaissance de l'espace, et comment obtenons-nous (ensemble) une connaissance de notre espace? L'explication psychophysiologique concerne les sensations et leurs associations, c'est une pièce de la psychologie associationniste. Poincaré développe l'idée selon laquelle les sensations, visuelles, tactiles, musculaires, ne nous donnent pas la notion d'espace directement, car, par elles-mêmes, elles n'ont pas de caractère spatial. Elles ne produisent pas la notion d'espace par une relation causale directe, comme une vue empiriste naïve pourrait le laisser croire. Nous n'avons pas de connaissance directe de l'espace physique. En fait, comme on l'a remarqué, l'expression "espace physique" ne fait pas partie du vocabulaire philosophique de Poincaré. De son point de vue, il y a un espace sensible ou représentatif construit à partir de sensations visuelles, tactiles, de mouvement, et par un processus de classification. Cependant, cet espace empirique n'a aucune des propriétés du véritable espace géométrique : il n'est pas infini (ou même non borné), ni homogène, ni isotrope. Or, il s'agit moins d'expliquer l'origine et les propriétés de l'espace empirique que celles de l'espace géométrique pur, espace conceptuel sur lequel nous raisonnons et que nous pouvons utiliser dans nos théories physiques. Poincaré considère cependant les sensations comme ayant une fonction significative dans la création et le développement de notre concept d'espace géométrique. En particulier, les sensations fournissent à notre esprit l'occasion de construire l'espace géométrique. Notre esprit forge une relation entre les sensations et l'espace géométrique par des actes de classification et d'analyse de certains changements de nos sensations.

Son hypothèse fondamentale est, on le sait, que tout ensemble de classes de déplacements forme un groupe, au sens mathématique. Pour Poincaré, la

4. Dans ses études de 1901-1903 sur la question de la théorie empirique et psychophysiologique des origines de la géométrie, E. Mach tiendra d'abord à se démarquer de Poincaré.

5. Poincaré traite souvent la question d'un point de vue a purement subjectiviste *. Il semble dire que tout sujet individuel crée ou pourrait créer ses propres idées d'espace et de géométrie spatiale. Cependant, il utilise le pluriel a nous " pour décrire comment nous les créons. Il tient compte des communications entre sujets dans la création de la géométrie spatiale, une géométrie fondée sur des conventions agréées en commun. Dans le long terme, il adopte plutôt une épistémologie et une ontologie « intersubjectivistes - (voir ses discussions ultérieures sur l'objectivité de la science, Valeur de la science, 1905, ch. X, XI : l'objectivité est ce qui est commun à la plupart des êtres pensants et pourrait l'ètre à tous). Dans ses derniers mémoires (1907, 1912), il semble renforcer cette position intersubjectiviste, pour finir par se rallier, semble-t-il, à une épistémologie * évolutionniste . 
condition que les déplacements forment un groupe est en géométrie absolument nécessaire, et le concept de groupe est le fondement de toute la géométrie. Il trouve une confirmation décisive de cette conviction dans les travaux de Lie sur les fondements de la théorie des groupes géométriques, objet alors d'une publication récente (l'étude monumentale de Lie et Engel est publié, en trois parties : 1886-1890-1893). La géométrie de l'espace "ordinaire "devient l'étude des propriétés formelles d'un certain groupe continu à six paramètres, le " groupe euclidien ${ }^{6}$ : nous imposons ce groupe à la nature, même si l'expérience nous guide dans le choix du groupe le meilleur pour cette application. Selon Poincaré, l'esprit a la capacité innée de construire des groupes continus - qui constituent une catégorie plus large que les groupes euclidiens - et d'en appliquer quelques-uns à l'expérience. Le concept de groupe abstrait est un élément a priori de notre entendement, qui garantit que la géométrie peut être une science exacte et certaine. C'est sur ce point que les vues de Poincaré s'opposent à celles de Kant : pour Poincaré, l'élément a priori, pertinent pour la géométrie spatiale, se trouve dans notre entendement, et non dans une forme de notre sensibilité?.

Peut-on, reprenant appui sur la doctrine kantienne, poser le problème de l'espace comme un problème transcendantal, c'est-à-dire essentiellement distinct de problèmes d'origine ou de genèse psychologique ? C'est le point qui inspire la réinterprétation récemment proposée du projet de l'esthétique kantienne que nous devons à G. Granger ${ }^{8}$.

Dans la Critique de la Raison Pure, l'Esthétique, Kant définit l'espace comme " une représentation (Vorstellung) nécessaire et a priori, qui fonde toutes les intuitions externes (Aüssere)..., une condition de possibilité des phénomènes (Erscheinungen) ఎ. Ainsi, l'espace est une représentation a priori, car elle est nécessaire, puisqu'elle sert de fondement à toutes les intuitions externes : je peux concevoir un espace sans objet, mais non des objets sans

6. Quelles sont les propriétés du groupe des déplacements spatiaux ? La réponse est fondée sur des arguments empiriques autant que mathématiques. Poincaré souligne qu'il étudie les propriétés formelles du groupe. Tout groupe isométrique aura les mèmes propriétés. Poincaré étudie donc réellement le groupe abstrait, bien que le groupe lui-méme s'adapte à une situation concrète. C'est l'époque où l'approche abstraite du groupe gagne du terrain chez les mathématiciens (Cayley, Frobenius, H. Weber, W. Burnside...). Poincaré se situe dans cette voie, quoiqu'il n'aille pas jusqu'à l'axiomatique.

7. Poincaré a joué son rôle dans la promotion du concept de groupe comme instrument d'unification : voir surtout son étude de 1908, L'avenir des mathématiques. On peut considérer sa philosophie de la géométrie, notamment telle qu'elle est développée dans l'article de 1898 , . On the Foundations of Geometry *, (The Monist 9, [1]), comme une expression philosophique de ce courant, et, plus spécifiquement, une traduction philosophique du travail de Lie pour fonder la géométrie à partir du concept de groupe continu. D'une part, à la fin de son étude, il se réfère directement au travail - fondationnel , de Lie. D'autre part, il se rattache implicitement aux solutions données par Lie au problème de Riemann-Helmoltz-Lie de l'espace, en considérant seulement les trois groupes continus à six paramètres de mouvements rigides : euclidien, de Lobatchevski et de Riemann (le * groupe riemannien * peut ètre, soit le groupe elliptique, soit le groupe sphérique), comme les représentations possibles de ces groupes de déplacements.

8. Dans des séminaires non encore publiés sur l'espace, tenus à Aix-en-Provence au cours des années 80 . 
espace. C'est une intuition pure ou une forme pure de la sensibilité (le “ sens") - la forme étant, dans l'objet tel qu'il se manifeste dans l'intuition empirique (ou donnée par la sensation), c'est-à-dire le phénomène, l'ensemble des rapports selon lesquels s'ordonne la matière, ou le divers, des sensations. Par le sens externe, distingué du sens intime, nous nous représentons les objets comme étant hors de nous et placés dans l'espace, où ils ont leur figure, leur grandeur, leurs rapports réciproques, déterminés ou déterminables. Ainsi la spatialité est essentiellement associée à l'objet, ou phénomène, extérieur, à la différence de l'objet du sens interne. D'autre part, clle est représentation, mais cette représentation est aussi, et même plutôt, condition de représentation. Si l'espace n'était pas intuition a priori, il serait impossible, d'après Kant (et c'est tout l'objet de l'exposition dite transcendantale du concept), d'expliquer la valeur nécessaire et a priori des propositions géométriques. Il y a bien des objets mathématiques obtenus par construction de concepts dans l'espace, des mathemata spatiaux, par exemple en géométrie, mais on ne peut dire cependant que l'espace lui-même soit objet de pensée : c'est toujours par l'intermédiaire de schèmes que les objets mathématiques sont déterminés comme étant de nature spatiale. Cette détermination de la spatialité comme forme des phénomènes extérieurs est indéniablement une limitation de la pensée kantienne, mais la question reste posée de savoir si l'analyse de l'Esthétique ne reste pas suffisamment forte pour constituer un point d'appui solide pour le traitement de la question.

Écartons d'abord une objection relative à une prétendue impossibilité d'une géométrie indépendante qui découlerait de la notion d'intuition formelle, introduite par Kant dans la deuxième édition de la Critique. La difficulté naîtrait de ce que, pour Kant, la condition de la constitution de l'espace comme objet de la représentation est en même temps la condition des objets d'expérience, à savoir l'unité transcendantale. Dès lors on aboutirait à l'alternative suivante : ou bien l'espace n'est rien de plus qu'une condition d'objets de l'expérience, et alors il n'est pas lui-même objet, objet d'une géométrie indépendante, mais il est forme du monde ; ou bien l'espace est constitué comme objet, comme intuition formelle se suffisant à lui-même, et il est objet d'une géométrie indépendante ; alors il est juxtaposé au monde. Il convient ici de lire attentivement les explications données par Kant de la notion d'intuition formelle et ses commentaires, notamment dans la note 26 de la deuxième édition.

Kant affirme que l'espace représenté comme objet, comme cela a lieu en géométrie, contient plus que la simple forme de l'intuition, mais encore l'intuition formelle qui résulte d'une synthèse, laquelle apporte l'unité au divers fourni a priori par la forme ${ }^{9}$. Pour Kant, pour que le divers de

9. Dans l'Esthétique, Kant a attribué cette unité à la sensibilité elle-mème pour montrer qu'il s'agit d'une unité antérieure à tout concept, bien qu'elle suppose une synthèse qui ne peut provenir que de la spontanéité de l'entendement. Mais comme, par cette synthèse, l'espace et le temps sont donnés ou constitués dans leur unité comme intuitions, l'unité appartient à 
l'intuition soit donné à la conscience, il ne suffit pas qu'il soit en nous, mais il faut qu'il soit constitué pour nous en une représentation de l'intuition, de façon à ce qu'il devienne conscient et objet d'intuition. Il faut donc distinguer la constitution de l'intuition de l'espace comme objet de l'intuition, et cette intuition même. Ainsi, bien que l'unité soit constituée par l'entendement qui l'a produite par la synthèse de l'homogène, elle appartient à l'intuition qui est constituée par là comme représentation - c'est pour cela que Kant l'a attribuée dans l'esthétique, à la sensibilité et non à l'entendement. Que cette synthèse soit difficile à concevoir, c'est tout le secret du schématisme : comment constituer une représentation d'un contenu intuitif par une synthèse d'agrégation, alors que la continuité exclut la composition? Cette représentation est bien une intuition et non un concept, puisque son objet est singulier, à savoir l'espace et le temps : il n'y a qu'un espace, qu'un temps, où le tout est donné avant les parties. L'appel à la synthèse n'explique pas la multiplicité pure, mais son organisation en objet intuitif pur (Fortschritte) ${ }^{1}$. Quant à l'intuition formelle, c'est celle qui se constitue par la synthèse de l'appréhension, de la reproduction et de la récognition dans la première édition, lorsque ces synthèses portaient sur le divers pur a priori ; ou encore, c'est la synthèse figurative de la deuxième édition. Donc, c'est l'entendement qui est l'instrument de cette synthèse et la règle, c'est l'agrégation.

Cette caractérisation ne rend pas impossible, comme certains commentateurs de Kant l'ont cru, une géométrie indépèndante. En fait, si l'on regarde de près les textes, on voit que la constitution de l'intuition formelle est requise comme condition préalable de la constitution de l'intuition empirique. Elle peut donc se constituer indépendamment en tant que préalable comme objet d'une science indépendante, tout en étant comme condition, forme du monde. Elle est une sorte de pré-objet à l'égard de l'objet de l'expérience qu'elle rend possible, et non point un objet superposé à lưi ${ }^{11}$. La mathématique n'est donc plus subordonnée à la physique dans son existence, mais seulement dans sa valeur objective. Car, si elle ne portait pas sur des conditions de l'expérience possible, et si elle ne devait pas se trouver ultérieurement réalisée dans l'expérience, elle resterait dépourvue de valeur objective, son objet resterait purement idéal et ne donnerait pas lieu à une connaissance véritable ; il n'en pourrait pas moins se constituer ${ }^{2}$.

À partir de là, et en prenant quelque distance d'avec le kantisme, on pourrait poser un premier problème, qui serait celui de savoir si la spatialité peut être objet d'une pensée en dehors du schème qui l'incarne. S'il en était

l'intuition a priori, mais non au concept de l'entendement.

10. Kant dit avoir attribué, dans l'Esthétique, cette unité à la sensibilité : il s'agit du troisième argument de l'espace, où l'espace est représenté comme une intuition pure et un objet singulier.

11. Ceci ne pourrait être soutenu, à certains égards, que dans la première édition

12 La deuxième édition insiste sur ce point que la condition de la connaissance ne consiste pas simplement dans la présence d'une intuition pure a priori, mais dans celle d'une intuition empirique ; c'est seulement de cette façon qu'on peut parler de subordination à la physique. 
ainsi, on pourrait entretenir l'espoir de concilier la spatialité comme schéma opératoire de la pensée objective avec la spatialité comme objet de pensée, ou encore la spatialité comme condition (de représentation d'objets) avec la spatialité comme objet même. Si on parvient à préciser la conception d'un espace-objet, et non simplement d'un espace-condition, on pourra du même coup renouveler la signification du kantisme. Dans la perspective de G. Granger, mettant au premier plan la dualité de l'objet et de l'opération, il faudrait alors toujours corréler l'espace avec des systèmes opératoires : la spatialité traduirait le schématisme kantien, mais un schématisme non autonome, lié à des systèmes d'opérations. L'espace serait pensable comme un méta-objet ${ }^{13}$ : un objet plus général et plus abstrait, ou un objet-forme, qui serait lui-même condition de représentation d'objets, de différents objets spécifiques. Le problème devient : comment déterminer ce qui, d'un système de formes abstraites (à la fois opératoires et objectales au sens de Granger), permet de définir une spatialité ? Il s'agit alors de déterminer des traits généraux communs (contenus formels, ou contenus de connaissance) qui se trouvent attachés à la spatialité comme méta-objet en général. G. Granger l'a posée sous la forme de celle de l'existence d'une spatialité naturelle, de la position possible d'un concept naturel d'espace. L'espace serait, avec le nombre, l'un des deux exemples privilégiés de “ concepts naturels ». On retrouverait bien ainsi la vue de Kant pour qui nombre et spatialité sont les deux sources de toute forme d'objet : un objet abstrait, a naturel ", mais non immédiatement réductible à l'espace usuel. Ce serait justement la tâche du philosophe de reconstituer, par le moyen d'une analyse des ceuvres de la mathématique, le concept naturel. Une telle tâche est bien différente de celle que lui assignait Husserl, puisqu'il ne s'agit pas de faire une phénoménologie, mais une épistémologie, articulée à l'histoire.

Quel est ici le sens de a naturel »? Il s'oppose tout à la fois à empirique et à mathématique. Un concept naturel c'est d'abord un concept transcendantal, et ceci en un double sens. D'abord en ce qu'il détermine a priori les cadres de toute pensée d'objet. L'espace naturel est source à la fois de l'espace perceptif et de l'espace mathématique, dans la mesure où, en tant que système des règles d'un univers symbolique, il est projetable dans le monde perceptif et mathématique. C'est en ce sens qu'on peut dire qu'il a une naturalité anté-prédicative. Ensuite en ce qu'il est synthétique (et donc synthétique a priort). Son unité synthétique provient de ce qu'il associe de façon nécessaire des propriétés non déductibles les unes des autres, qu'il réalise la synthèse entre des éléments ensuite dissociés par l'activité mathématique, aux prises avec des contraintes et des obstacles nés précisément de ce caractère synthétique, de cette solidarité des éléments constitutifs du concept, qui s'oppose à la mise en évidence de ces éléments, à

13. Un méta-objet est un objet relié non directement à une expérience, mais à une représentation de l'expérience, et objet d'une méta-discipline. 
la dissociation de ces concepts naturels en concepts partiels. Il y a ici une intrication essentielle, une inséparabilité des traits ultérieurement dégagés, par exemple algébrique et différentielle (exemple de l'analyse tensorielle, l'algèbre multilinéaire), ou algébrique et topologique (exemple de la théorie homologique de la dimension), et c'est ce qui explique que, même dans la pure mathématique, l'idée d'espace a une consistance et exige la multiplication des points de vue ${ }^{14}$.

Le concept naturel est la synthèse de ces caractères dissociés - synthèse a priori en ceci que les concepts mathématiques produits (les éléments mathématiques dissociés\} fonctionnent ici comme cadre d'une pensée d'objets, comme " guides transcendantaux d'une pensée d'objets - d'où découle leur caractère opératoire ou instrumental - et synthèse originaire, puisque tel est justement le trait qui distingue les concepts naturels des autres concepts. Dans cette perspective, c'est l'œuvre mathématique ellemême qui fournit, directement, à la réflexion les matériaux conceptuellement élaborés qu'elle se donne pour tâche d'interpréter. Si les concepts naturels existent, c'est à la philosophie de les reconnaître, mais, en retour, il faut dire que seul le travail conceptuel du mathématicien peut mettre le philosophe en état de reconnaître et de formuler les éléments du complexe dont il essaie de mettre en évidence le caractère synthétique ${ }^{15}$. Ainsi peut-on comprendre que le travail des mathématiciens élabore des concepts propres à donner de ce monde une image indépendante des formes mèmes de l'intuition, à dessiner une spatialité conceptuelle, et non intuitive, et à nous obliger en fin de compte à repenser la notion même d'objet. La norme de spatialité, si elle peut être comprise en elle-même comme une organisation de structures abstraites, elles-mêmes dissociables en sous-structures, donne en effet des contraintes plus générales que les contraintes empiriques, et révèle la possibilité d'un usage physique de la spatialité du mathématicien pour dépasser le donné immédiat. L'“ espace de Hilbert „ donne l'exemple d'une structuration mathématique très éloignée de notre échelle : il peut y avoir une infinité de dimensions. On obtient un concept très différent, à la fois de l'espace euclidien et de l'espace non euclidien, et pourtant on continue d'appeler une telle structure " espace ", et cet espace a un usage physique, il sert au physicien à une autre échelle. D'où l'on comprend peut-être en quoi on peut penser les objets autrement, en quoi par exemple les objets microphysiques ne s'insèrent pas plus dans le cadre d'une spatialité que les nombres complexes ne s'insèrent dans le cadre des nombres naturels. Finalement, on pourrait fort bien de cette manière restituer le thème kantien de l'esthétique,

14. L’unité synthétique du concept naturel se manifeste par la présence de contenus formels : c'est-à-dire de corrélations d'opérations et d'objets, critère qui vient se substituer au critère kantien de référence au sensible, du moins à ses formes a priori.

15. Granger note qu'il y a deux aspects, opposés et complémentaires, du travail du mathématicien: l'un de décomposition ou dissociation, l'autre de restitution ou reconstitution, des objets naturels : voir Sur l'idée de concept mathématique naturel, 1.1 (Formes, opérations, objets, Paris, 1994, p. 158-159). 
en dégageant, à partir du travail des mathématiciens, une a esthétique transcendantale pour des mondes sensibles imaginaires ${ }^{16} \mathrm{~m}$.

Le problème fondamental est alors le suivant : quel jeu de propriétés opératoires d'un système abstrait se trouve être corrélatif d'une objectité spatiale? Et comment ces propriétés se trouvent-elles déterminer de manière analytico-algébrico-géométrique des formes d'objets empiriques imaginaires? On retrouverait là le plan où s'est placé $\mathbf{M}$. Boi dans son livre, par exemple lorsqu'il distingue, au début, quatre sens du mot espace : sensible (ou physiologique, ou perceptif), intuitif, mathématique, physique. Dans la perspective de G. Granger, l'espace intuitif est remplacé par l'espace naturel, c'est-à-dire le concept naturel d'espace : signe qu'on s'est écarté d'une référence à la phénoménologie, pour rapprocher le travail épistémologique de l'histoire, entendu comme analyse des œuvres. Trois conditions sont proposées pour une pensée minimale de la spatialité : la dimensionnalité, l'opposition du local et du global, la possibilité de mesures ${ }^{17}$. On retrouverait assez facilement, dans les méandres du cheminement historique que suit l'ouvrage de M. Boi, les deux derniers points de vue. Le premier est moins présent, mais il est lié en profondeur à la notion de forme, sur laquelle l'auteur nous promet une étude ultérieure ${ }^{18}$. Des deux grands modes de saisie opératoire de constitution de la forme, la première, comme invariant d'un groupe de transformations, est abondamment traitée, puisque c'est l'exemple même des géométries non euclidiennes qui a tout à la fois révélé l'existence des formes et permis de libéraliser l'invariant - les formes étant déterminées par le choix d'une configuration fondamentale (c'est-à-dire transformée en elle-même par les transformations du groupe) absolument invariante. La structure globale d'un espace détermine alors une famille de formes, rigidement associées à une forme éponyme. On obtient plusieurs espaces différents, associées à des groupes différents de transformations et à des propriétés invariantes par ces groupes. La seconde, qui serait la saisie de la forme comme combinatoire sur des éléments, dans la topologie combinatoire et algébrique, n'est pratiquement pas envisagée dans l'ouvrage. L'auteur relève (p. 103) qu'une même géométrie locale peut être compatible avec des structures globales très

16. C'était déjà un projet de Husserl (voir Logique formelle et logique transcendantale, conclusion, et le commentaire de Cavaillès dans Sur la logique..., p. 61-ss). Husserl y affirme, en effet, la nécessité d'une esthétique transcendantale élargie qui traite le problème d'un monde possible en général comme monde de pure expérience, antérieurement à toute activité catégorielle et définisse les conditions a priori de l'unité propre pouvant lui être attribuée, et qui servira de base aux enchainements apophantiques - thème de la logique. C'est une conséquence de la doctrine de la * relevance des noyaux * : les matériaux syntaxiques doivent avoir affaire entre eux matériellement, par la solidarité interne des contenus qu'ils relient. On ne peut juxtaposer n’importe quelles syntaxes : le mode de jugement originel est le jugement évident, fondé sur l'expérience : antérieurement à toute action du juger, se trouve le sol universel de l'expérience, constamment supposé comme unité d'expérience possible. On ne peut partir du logique pur: la légitimation de l'unité apophantique est l'affinité préalable des contenus expérimentaux qu'elle organise.

17. Sur lidée de concept mathématique naturel, 2.4. (op. cit., p. 174 et ss.)

18 Ibid., p. 351, note 40. 
différentes, comme le montre l'exemple du cylindre (ou du cône, sauf au point singulier) et du plan qui sont localement isométriques, et dont la géométrie est donc identique localement, quoique globalement différente. Selon F. Klein, la géométrie locale ne détermine pas univoquement la globale. Le « prolongement géométrique o d'une métrique donnée, différent en cela du prolongement analytique, n'est pas univoque : on peut construire différentes structures globales d'espace (Raumformen). Mais on sait aujourd'hui représenter des structures topologiques globales. On sait mème représenter, dans l'espace euclidien, des structures qui sont aberrantes pour cet espace. Mettant en évidence des points de repère (d'où la nécessité d'un repérage) arbitraires, et faisant jouer sur ces points de repère des opérations, du type flèches, on définit une géométrie globale sur une région spatiale en la recouvrant d'un réseau de morceaux d'espace élémentaires, du type polyèdres, qui permettent de faire de la topologie combinatoire ou algébrique. Les systèmes opératoires déterminent alors des objets très généraux et invariants, par généralisation de la procédure qui consistait à attacher des nombres à des grandeurs géométriques : ici, les invariants ne sont pas seulement numériques, mais peuvent aussi bien être des groupes. On se retrouve dans un chapitre d'algèbre générale, et encore au seuil d'une étude de la spatialité. Le privilège est donné à une famille de concepts préspatiaux, comme celui de figure : une figure géométrique, réalisée sur une certaine forme de spatialité, est déterminée par un nombre fini de bords. Il s'agit d'une pré-géométrie, qui prend pour objet la relation de la figure à un champ opératoire. L'objet de l'homotopie est différent : il s'agit de savoir quelles déformations des figures sont autorisées par l'espace où elles sont plongées. Le problème est celui du rapport des figures à un espace, et l'espace intervient comme un milieu distinct des figures, où il y peut y avoir des obstacles aux déformations. Les considérations introduites sont " texturales ", et le concept de spatialité doit avoir cette double structuration de la forme et de la texture. Sans doute, une telle description reste par ellemême insuffisante, et la spatialité n'est introduite que par la dimension, en tant qu'on sait la lier à une mesure, c'est-à-dire un contenu de grandeur : en retrouvant par exemple, dans un cadre adéquat, la vieille idée grecque de différence entre ligne et surface comme dans la mesure p-dimensionnelle d'un ensemble de Hausdorff. Mais les opérations de la topologie algébrique procurent la structuration préalable nécessaire à cet investissement effectif de la mesure. Cet aspect reste quelque peu dans l'ombre dans l'ouvrage de M. Boi, et la raison serait peut-être à chercher dans une certaine sousestimation du rôle de l'algèbre. Il est arrivé à Claude Chevalley d'affirmer que l'algèbre est, beaucoup plus qu'une partie des mathématiques, la discipline mère, jouant dans les mathématiques le rôle de la physique par rapport aux 
mathématiques elles-mêmes. Cette précellence de l'algèbre était un trait essentiel, et une des origines, du bourbakisme. La « quête de l'intelligible nà laquelle nous convie L. Boi ne vise qu'à restituer l'hégémonie à la géométrie.

Département de philosophie

Université de Provence 